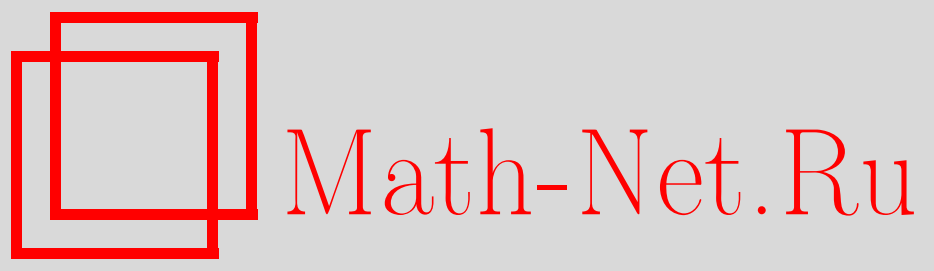

М. И. Кацнельсон, В. Е. Назайкинский, Эффект Ааронова-Бома для безмассовых дираковских фермионов и спектральный поток операторов типа Дирака с классическими граничными условиями, ТМФ, 2012, том 172, номер 3, 437-453

DOI: https://doi.org/10.4213/tmf8337

Использование Общероссийского математического портала Math-Net.Ru подразумевает, что вы прочитали и согласны с пользовательским соглашением http: //www . mathnet.ru/rus/agreement

Параметры загрузки:

IP : 18.207 .199 .55

26 апреля 2023 г., 13:34:30

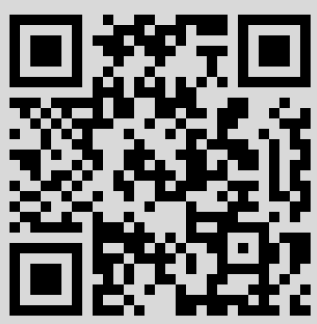




\title{
ЭФФЕКТ ААРОНОВА-БОМА ДЛЯ БЕЗМАССОВЫХ ДИРАКОВСКИХ ФЕРМИОНОВ И СПЕКТРАЛЬНЫЙ ПОТОК ОПЕРАТОРОВ ТИПА ДИРАКА С КЛАССИЧЕСКИМИ ГРАНИЧНЫМИ УСЛОВИЯМИ
}

\begin{abstract}
В топологических терминах вычислен спектральный поток произвольного семейства самосопряженных операторов типа Дирака с классическими (локальными) граничными условиями на компактном римановом многообразии с краем в предположении, что начальный и конечный операторы семейства сопряжены с помощью некоторого автоморфизма расслоения, в котором они действуют. С помощью этого результата исследованы условия существования ненулевого спектрального потока семейства самосопряженных операторов типа Дирака с локальными граничными условиями в двумерной области с нетривиальной топологией. Обсуждаются возможные физические реализации ненулевого спектрального потока.
\end{abstract}

Ключевые слова: эффект Ааронова-Бома, безмассовые дираковские фермионы, графен, топологические изоляторы, самосопряженный оператор Дирака, классическая краевая задача, спектральный поток, теорема Атьи-Зингера об индексе, теорема Атьи-Ботта об индексе, принцип локальности индекса.

\section{1. ВВЕДЕНИЕ}

Вслед за физикой высоких энергий и квантовой теорией поля в физике конденсированного состояния все активнее используются идеи современной геометрии и топологии [1]-[9]. В частности, теорема Атьи-Зингера об индексе [10] объясняет топологическую устойчивость нулевого уровня Ландау и аномалии квантового эффекта Холла в графене [6], [7]. Топологически защищенные нулевые моды играют существенную роль в движении вихрей в сверхтекучем $\mathrm{He}^{3}$ [5], [11]. Квантовый эффект

*Institute for Molecules and Materials, Radboud University Nijmegen, Nijmegen, The Netherlands. E-mail: m.katsnelson@science.ru.nl

${ }^{\dagger}$ Институт проблем механики им. А. Ю. Ишлинского РАН, Москва, Россия. E-mail: nazay@ipmnet.ru

${ }^{\ddagger}$ Московский физико-технический институт, г. Долгопрудный, Московская обл., Россия

$\S$ Московский государственный институт электроники и математики, Москва, Россия 
Холла [2], [3] и топологические изоляторы [8], [9] представляют собой особые состояния с топологическим параметром порядка.

Открытие эффекта Ааронова-Бома (ЭАБ) [12], [13], в сущности, положило начало этим исследованиям. Поток магнитного поля, локализованный в области, полностью недоступной для квантовой частицы (например, окруженный бесконечно высоким барьером), влияет, тем не менее, на ее движение, изменяя геометрию квантового пространства. Ярким проявлением ЭАБ является периодическая зависимость энергетических уровней электронов в кольце от магнитного потока через кольцо, приводящая к появлению спонтанного недиссипативного тока (см., например, работу [14] и цитированную в ней литературу). Когда поток меняется на целое число квантов потока, энергетический спектр системы должен вернуться в исходное состояние. Вплоть до недавнего времени основное внимание уделялось ЭАБ для обычных нерелятивистских электронов, описываемых уравнением Шредингера.

Недавно ЭАБ для ультрарелятивистских электронов, описываемых уравнением Дирака с нулевой массой, привлек внимание в связи с исследованиями графена [15]-[17]. С математической точки зрения это гораздо более богатая ситуация. Оператор Дирака не является полуограниченным, и потому, вообще говоря, его спектральный поток [18] может быть отличен от нуля. Отметим, что ненулевой спектральный поток оператора Дирака уже обсуждался в контексте физики конденсированного состояния. Именно он приводит к появлению дополнительных сил ("сил Копнина"), действующих на вихри в сверхтекучем $\mathrm{He}^{3}$ [5], [11]. В случае ЭАБ это означает, что возвращение энергетического спектра в целом к исходному через целое число квантов потока не обязательно предполагает периодичность каждого собственного значения по отдельности. Ненулевой спектральный поток физически соответствует ситуации, когда адиабатически медленно меняющееся магнитное поле приводит к рождению электронно-дырочных пар (в более общих терминах - пар частица-античастица) из вакуума: "позитронные" уровни пересекают линию нулевой энергии, превращаясь в электронные. Эффекты "перестройки вакуума" обсуждались в физике сверхтекучего $\mathrm{He}^{3}$ [5] и в физике графена [7], но вне всякой связи с ЭАБ. В настоящей работе мы исследуем условия существования ненулевого спектрального потока семейства самосопряженных операторов типа Дирака с локальными граничными условиями в области с нетривиальной топологией.

В ограниченной области $X \subset \mathbb{R}^{2}$ с гладкой границей $\partial X$ (см. рис. 1 ) рассмотрим краевую задачу

$$
\left(\begin{array}{cc}
0 & -i \frac{\partial}{\partial x}-\frac{\partial}{\partial y} \\
-i \frac{\partial}{\partial x}+\frac{\partial}{\partial y} & 0
\end{array}\right)\left(\begin{array}{l}
u_{1} \\
u_{2}
\end{array}\right) u=\left(\begin{array}{l}
f_{1} \\
f_{2}
\end{array}\right) \text { в } X, \quad\left(n_{y}-i n_{x}\right) u_{1}=B u_{2} \text { на } \partial X
$$

где $n_{x}, n_{y}$ - компоненты внутренней нормали, а $B$ - не обращающаяся в нуль вещественная функция на границе (краевые условия такого рода впервые рассматривались Берри и Мондрагоном [19]). Соответствующий этой задаче оператор $D_{0}$ самосопряжен и фредгольмов в $L^{2}\left(X, \mathbb{C}^{2}\right)$. Далее, пусть $\mu$ - гладкая функция в области $X$ и $|\mu|=1$. "Калибровочное преобразование" $D_{0} \rightarrow \mu D_{0} \mu^{-1}$ переводит оператор $D_{0}$ в оператор $D_{1}=D_{0}+Q_{1}$ (где $Q_{1}$ - самосопряженная матричная функция) с теми же граничными условиями. В физических терминах градиент фазы $\mu$ есть абелево $(U(1))$ калибровочное поле - электромагнитный векторный потенциал. Мы рассматриваем двумерную область с $m-1$ дырками, пронизанными трубками магнитного 


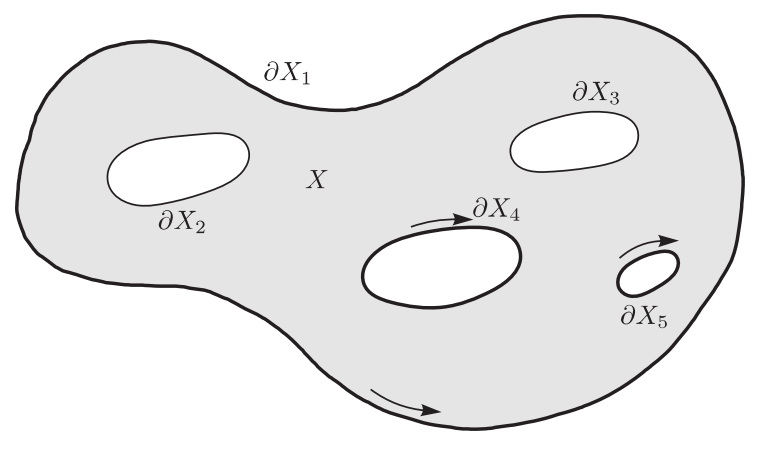

Рис. 1. Пример области $X$ с $m=5$ компонентами границы. Жирными линиями выделены компоненты границы $\partial^{+} X=\partial X_{1} \cup \partial X_{4} \cup \partial X_{5}$, на которых $B>0$ в краевом условии из задачи (1) (только эти компоненты и дают вклад в спектральный поток согласно формуле (3)). Стрелки указывают положительное направление обхода при вычислении числа вращения калибровочного преобразования $\mu$ вдоль соответствующей компоненты границы.

поля. Случай, когда все магнитные потоки через дырки равны целому числу квантов потока, соответствует значению $\mu=1$. Соединим $Q_{1}$ с $Q_{0}=0$ непрерывным семейством $Q_{t}, t \in[0,1]$, самосопряженных матричных функций. Спектральный поток $\operatorname{sf}\left\{D_{t}\right\}$ семейства

$$
D_{t}=D_{0}+Q_{t}
$$

- число собственных значений оператора $D_{t}$, изменивших знак с минуса на плюс при изменении параметра $t$ от нуля до единицы, минус число собственных значений, изменивших знак с плюса на минус, - не меняется при непрерывных деформациях семейства, не нарушающих изоспектральности операторов $D_{0}$ и $D_{1}$. Насколько известно авторам, задача о вычислении этого спектрального потока (также и для операторов Дирака более общего вида) была впервые поставлена и частично решена Прохоровой [20], которая вычислила спектральный поток с точностью до целочисленного множителя $c_{m}$, зависящего от числа $m$ компонент границы. Далее, в [20] было показано, что $c_{2}=1$, и была выдвинута гипотеза, что $c_{m}=1$ для всех $m$. В настоящей работе мы устанавливаем общий результат (см. ниже теорему 2), из которого, в частности, вытекает справедливость этой гипотезы. Таким образом, теорема 1 работы [20] приобретает следующий вид.

Теорема 1. Спектральный поток семейства (2) можно вычислить по формуле

$$
\operatorname{sf} D_{t}=\operatorname{wind}_{\partial^{+} X} \mu
$$

где $\partial^{+} X-$ та часть границы $\partial X$, на которой $B>0, a$

$$
\underset{\partial^{+} X}{\operatorname{wind}} \mu=\frac{1}{2 \pi i} \oint_{\partial^{+} X} \frac{d \mu}{\mu}
$$

- число вращения ограничения функиии $\mu$ на $\partial^{+} X$. (Множество $\partial^{+} X$ является объединением конечного числа окружностей; положителъным направлением при 
определении числа вращения считается то, при котором область $X$ остается слева по ходу движения.)

Эта теорема показывает, что коэффициенты $c_{m}$, оставшиеся невычисленными в теореме 1 работы [20], равны единице при всех $m$. То же самое относится и к теоремам 2 и 3 упомянутой работы - фигурирующие там неопределенные коэффициенты $c_{m}$ равны единице.

Теорема 1 вытекает из установленного здесь общего результата: мы вычисляем в топологических терминах спектральный поток произвольного семейства $\left\{D_{t}\right\}$, $t \in[0,1]$, самосопряженных операторов типа Дирака с локальными граничными условиями на компактном римановом многообразии $X$ с краем $\partial X$ в предположении, что $D_{1}=U D_{0} U^{-1}$, где $U-$ некоторый автоморфизм расслоения, в котором действуют операторы $D_{t}$. (При этом, в отличие от [20], мы не предполагаем, ни что главная часть оператора $D_{t}$ не зависит от $t$, ни даже что главные части операторов $D_{0}$ и $D_{1}$ совпадают.) Именно, мы доказываем (см. ниже теорему 2), что

$$
\operatorname{sf}\left\{D_{t}\right\}=\operatorname{ind}\left(\frac{\partial}{\partial t}+D\right),
$$

где в правой части стоит индекс эллиптического оператора с граничными условиями на многообразии $X \times S^{1}$ с краем, причем $t$ - координата на окружности $S^{1}$, а оператор $D$ получается из семейства $\left\{D_{t}\right\}$ сцеплением операторов $D_{0}$ и $D_{1}$ по автоморфизму $U$ (напомним, что равенство (4) для семейств самосопряженных эллиптических операторов на замкнутом многообразии $X$ установлено в [18]). Правая часть равенства (4) может быть вычислена по формуле Атьи-Ботта [21] (см. также [22], раздел 20.3).

Заметим, однако, что в доказательстве теоремы 1 формулу Атьи-Ботта мы не используем: равенство (4) спектрального потока индексу позволяет использовать метод локализации (см. [23]-[25]) и, разрезая область на части, свести дело к случаю области с одним отверстием $(m=2)$, для которого в работе [20] проделано явное вычисление. Отметим также, что метод локализации оказывается важным техническим инструментом и при доказательстве самого равенства (4), которое во многом аналогично доказательству (см. [26], предложение 5.6) формулы спектрального потока для эллиптических с параметром в смысле Аграновича-Вишика семейств дифференциальных операторов на замкнутом многообразии без края, но содержит ряд новых моментов, связанных с наличием граничных условий.

\section{2. СПЕКТРАЛЬНЫЙ ПОТОК}

Напомним определение спектрального потока в форме, приведенной в [26] (см. также [27]). Пусть $\left\{B_{t}\right\}, t \in[0,1],-$ непрерывное в смысле равномерной резольвентной сходимости семейство неограниченных самосопряженных операторов с чисто дискретным спектром в гильбертовом пространстве $\mathcal{H}$. Тогда существуют разбиение $0=t_{0}<t_{1}<t_{2}<\cdots<t_{n+1}=1$ отрезка $[0,1]$ и вещественные числа $\gamma_{1}, \ldots, \gamma_{n+1}$ такие, что точка $\gamma_{j}$ не лежит в спектре $\operatorname{Spec}\left(B_{t}\right)$ оператора $B_{t}$ при $t \in\left[t_{j-1}, t_{j}\right]$, $\gamma_{1}=\gamma_{n+1} \leqslant 0$, и если $\gamma_{1}<0$, то полуинтервал $\left[\gamma_{1}, 0\right)$ не содержит точек спектра операторов $B_{0}$ и $B_{1}$. 


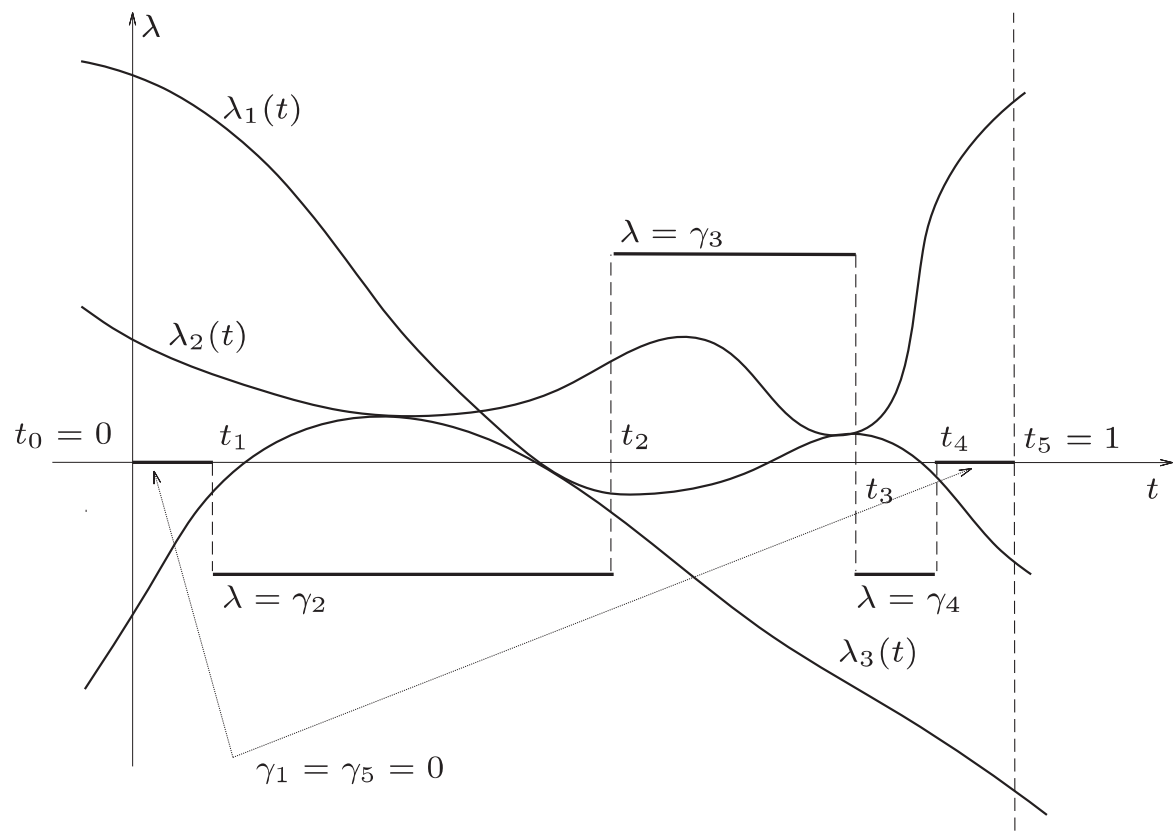

Рис. 2. Определение спектрального потока. Вклад в спектральный поток дают три собственных значения $\lambda_{j}(t), j=1,2,3$. И вычисление в соответствии с определением, и подсчет числа прохождений (с учетом направления) через нуль дают для спектрального потока значение -1 .

ОПРЕДЕЛЕНИЕ 1 (см. [26], определение А.18). Спектралъным потоком семейства $\left\{B_{t}\right\}, t \in[0,1]$, называется число ${ }^{1)}$

$$
\operatorname{sf}\left\{B_{t}\right\}=\sum_{j=1}^{n} m_{j} \operatorname{sign}\left(\gamma_{j}-\gamma_{j+1}\right),
$$

где $m_{j}$ - количество собственных значений (с учетом кратности) оператора $B_{t_{j}}$ в интервале между $\gamma_{j}$ и $\gamma_{j+1}$.

Это определение проиллюстрировано на рис. 2, из которого, помимо всего прочего, становится ясно, почему это определение согласуется с определением спектрального потока как числа прохождений собственных значений через нуль (с учетом направления).

\section{3. ОСНОВНЫЕ РЕЗУЛЬТАТЫ}

Пусть $E$ - четномерное эрмитово векторное расслоение над компактным римановым многообразием $X$ с краем, и пусть

$$
A: C^{\infty}(X, E) \longrightarrow C^{\infty}(X, E)
$$

1) Правая часть формулы (5) не зависит от выбора разбиения $\left\{t_{j}\right\}$ и чисел $\gamma_{j}$ согласно теореме А.19 из [26]. 
- формально самосопряженный оператор типа Дирака ${ }^{2)}$. Далее, пусть в сужении $E_{Y}$ расслоения $E$ на край $Y=\partial X$ многообразия $X$ задано подрасслоение $L \subset E_{Y}$ размерности $\operatorname{dim} L=(\operatorname{dim} E) / 2$ такое, что

$$
\left(\sigma_{A}(x, \mathbf{n}(x)) L_{x}\right) \perp L_{x} \quad \forall x \in Y,
$$

где $L_{x}$ - слой расслоения $L$ в точке $x$, а $\mathbf{n}(x)$ - единичный вектор внутренней конормали к границе. Рассмотрим оператор (6) на множестве сечений $u \in C^{\infty}(X, E)$, удовлетворяющих однородному граничному условию

$$
\pi_{L}\left(\left.u\right|_{Y}\right)=0, \quad \text { где } \pi_{L}: E_{Y} \longrightarrow E_{Y} / L \quad \text { - естественная проекция }
$$

(иными словами, $u(x) \in L_{x}$ при $x \in Y$ ). Такой вид имеет, в частности, граничное условие в задаче (1). Хорошо известно (см. [29], а также гл. 18 и 19 в [28]), что граничное условие (8) является эллиптическим, оператор (6) с областью определения, задаваемой этим условием, существенно самосопряжен в $L^{2}(X, E)$, а его замыкание $A_{L}$ представляет собой неограниченный фредгольмов самосопряженный оператор в $L^{2}(X, E)$ с дискретным спектром и с областью определения, состоящей из сечений $u$ из пространства Соболева $H^{1}(X, E)$, удовлетворяющих условию (8) (в котором $\left.u\right|_{Y}$ понимается как элемент пространства Соболева $H^{1 / 2}\left(Y, E_{Y}\right.$ ), полученный из $u$ ограничением на $Y$ в силу теоремы о следах, а $\pi_{L}$ - как отображение $\left.\pi_{L}: H^{1 / 2}\left(Y, E_{Y}\right) \longrightarrow H^{1 / 2}\left(Y, E_{Y} / L\right)\right)$.

Пусть теперь и оператор $A$ типа Дирака (6), и подрасслоение $L$ непрерывно зависят от параметра $t \in[0,1]$ (а именно, коэффициенты оператора $A$ и оператора $\pi_{L}$ зависят от $t$ непрерывно вместе со всеми своими производными $\left.{ }^{3)}\right)$, т. е. $A=A(t)$, $L=L(t)$, причем при каждом $t$ выполнено условие $(7)$. Тогда по теореме 7.16 из работы [30] оператор $A(t)_{L(t)}$ непрерывно зависит от $t$ в топологии равномерной резольвентной сходимости, и определение 1 задает спектральный поток $\operatorname{sf}\left\{A(t)_{L(t)}\right\}$ семейства $\left\{A(t)_{L(t)}\right\}, t \in[0,1]$.

Далее, пусть задан автоморфизм $U: E \rightarrow E$ расслоения $E$ такой, что

$$
A(1)=U A(0) U^{-1}, \quad U(L(0))=L(1) .
$$

Тогда $U A(0)_{L(0)} U^{-1}=A(1)_{L(1)}$, т. е. операторы $A(0)_{L(0)}$ и $A(1)_{L(1)}$ подобны и, следовательно, изоспектральны, так что спектральный поток семейства $\left\{A(t)_{L(t)}\right\}$ является гомотопическим инвариантом (в классе семейств, удовлетворяющих условию вида (9)), и поэтому естественно поставить задачу о его вычислении в топологических терминах.

Чтобы решить эту задачу, введем вспомогательную эллиптическую краевую задачу на прямом произведении $X \times S^{1}$ многообразия $X$ на окружность $S^{1}$ (см. рис. 3 ). Именно, определим расслоение $\mathcal{E}$ на $X \times S^{1}$, поднимая расслоение $E$ на произведение

2) Напомним, что линейный дифференциальный оператор первого порядка (6) называется оператором типа Дирака, если его главный символ $\sigma_{A}(x, \xi)$ удовлетворяет условию $\left(\sigma_{A}(x, \xi)\right)^{2}=$ $\sum g^{j k}(x) \xi_{j} \xi_{k} I$, где $I$ - тождественный оператор в слое $E_{x}$, а $g^{j k}(x)$ - (контравариантные) компоненты метрического тензора (см. [28]). Формальная самосопряженность оператора $A$ понимается в стандартном смысле как выполнение тождества $(u, A v)=(A u, v)$ для любых сечений $u, v \in C_{0}^{\infty}(X \backslash \partial X, E)$, где $(\cdot, \cdot)$ - скалярное произведение в $L^{2}(X, E) \equiv L^{2}(X, E, d$ vol $)$. 3десь $d$ vol элемент риманова объема на многообразии $X$.

3) Достаточно, судя по всему, и одной производной, но не будем мелочиться. 


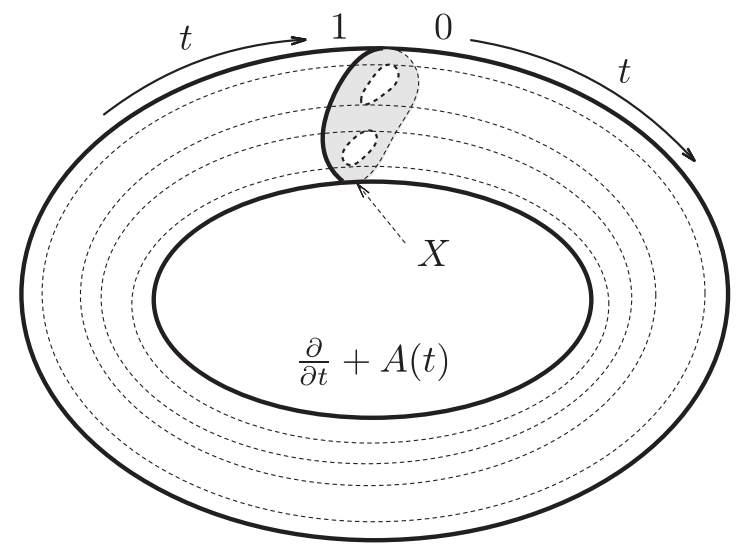

Рис. 3. Многообразие $X \times S_{1}$, полученное склейкой "торцов" $t=0$ и $t=1$ произведения $X \times[0,1]$, с оператором $\partial / \partial t+A(t)$.

$X \times[0,1]$ с помощью естественной проекции $\pi: X \times[0,1] \rightarrow X$ и затем производя сцепление ${ }^{4)}$ по автоморфизму $U:\left(\pi^{*} E\right)_{X \times\{0\}} \longrightarrow\left(\pi^{*} E\right)_{X \times\{1\}}$. В силу условий (9) семейство $\{A(t)\}$ корректно определяет дифференциальный оператор в пространстве сечений расслоения $\mathcal{E}$, а семейство подрасслоений $L(t)$ определяет подрасслоение $\mathcal{L} \subset \mathcal{E}_{Y \times S^{1}}$ в сужении расслоения $\mathcal{E}$ на край $Y \times S^{1}$ многообразия $X \times S^{1}$.

ПредлОЖЕНИЕ 1. Оператор

$$
\frac{\partial}{\partial t}+A(t): C^{\infty}\left(X \times S^{1}, \mathcal{E}\right) \longrightarrow C^{\infty}\left(X \times S^{1}, \mathcal{E}\right)
$$

эллиптический, и граничнье условия

$$
\pi_{\mathcal{L}}\left(\left.u\right|_{Y \times S^{1}}\right)=0, \quad \text { где } \pi_{\mathcal{L}}: \mathcal{E}_{Y \times S^{1}} \longrightarrow \mathcal{E}_{Y \times S^{1}} / \mathcal{L}-\text { естественная проекиия, }
$$

задаваемъе подрасслоением $\mathcal{L}$, являются для оператора (10) эллиптическими. Замыкание $(\partial / \partial t+A(t))_{\mathcal{L}}$ оператора $(10)$ с области, задаваемой условиями $(11)$, представляет собой неограниченный фредгольмов оператор в $L^{2}\left(X \times S^{1}, \mathcal{E}\right)$ с областъю определения $\mathfrak{D}_{\mathcal{L}}$, состоящей из сечений $u \in H^{1}\left(X \times S^{1}, \mathcal{E}\right)$, удовлетворяющих условию (11).

Теперь мы можем сформулировать основное утверждение настоящей работы.

Теорема 2. Справедлива формула

$$
\operatorname{sf}\left\{A(t)_{L(t)}\right\}=\operatorname{ind}\left(\frac{\partial}{\partial t}+A(t)\right)_{\mathcal{L}} .
$$

Правая часть формулы (12) представляет собой аналитический индекс оператора $(\partial / \partial t+A(t))_{\mathcal{L}}$, т. е. разность размерностей его ядра и коядра, который может быть выражен в топологических терминах по формуле Атьи-Ботта [21] (см. также [22], раздел 20.3).

\footnotetext{
4) Мы считаем, что окружность $S^{1}$ получена из отрезка $[0,1]$ склеиванием концов.
} 


\section{4. ДОКАЗАТЕЛЬСТВА ОСНОВНЫХ УТВЕРЖДЕНИЙ}

ДокАЗАТЕЛЬСтво ПРЕДЛОЖЕНИЯ 1. Рассмотрим оператор

$$
\mathfrak{A}=\left(\begin{array}{cc}
0 & \frac{\partial}{\partial t}+A(t) \\
-\frac{\partial}{\partial t}+A(t) & 0
\end{array}\right): C^{\infty}\left(X \times S^{1}, \mathcal{E} \oplus \mathcal{E}\right) \longrightarrow C^{\infty}\left(X \times S^{1}, \mathcal{E} \oplus \mathcal{E}\right) .
$$

Это “полный" формально самосопряженный оператор типа Дирака на $X \times S^{1}$ с символом

$$
\sigma_{\mathfrak{A}}\left(x, t, \xi, \xi_{0}\right)=\left(\begin{array}{cc}
0 & i \xi_{0} I+\sigma_{A(t)}(x, \xi) \\
-i \xi_{0} I+\sigma_{A(t)}(x, \xi) & 0
\end{array}\right),
$$

где $\xi_{0}$ - переменная, двойственная к $t \in S^{1}$, и оператор $\partial / \partial t+A(t)$ является его киральной частью. Подрасслоение $\mathfrak{L}=\mathcal{L} \oplus \mathcal{L} \subset(\mathcal{E} \oplus \mathcal{E})_{Y \times S^{1}}$ удовлетворяет условию вида (7) относительно $\sigma_{\mathfrak{A}}$ и тем самым задает самосопряженные эллиптические условия для $\mathfrak{A}$. Действительно, вектор конормали к границе многообразия $X \times S^{1}$ в произвольной точке $(x, t) \in Y \times S^{1}$ имеет вид $\mathfrak{n}(x, t)=(0, \mathbf{n}(x))$, где $\mathbf{n}(x)$ - вектор конормали к границе самого многообразия $X$, а $\mathcal{L}_{(x, t)}=L(t)_{x}$, поэтому для любых

$$
v={ }^{t}\left(v_{1}, v_{2}\right) \in \mathfrak{L}_{(x, t)}, \quad w={ }^{t}\left(w_{1}, w_{2}\right) \in \mathfrak{L}_{(x, t)}, \quad \text { т. е. } \quad v_{1}, v_{2}, w_{1}, w_{2} \in L(t)_{x},
$$

имеем

$$
\left(v, \sigma_{\mathfrak{A}}(x, t, \mathfrak{n}(x, t)) w\right)=\left(v_{1}, \sigma_{A(t)}(x, \mathbf{n}(x)) w_{2}\right)+\left(v_{2}, \sigma_{A(t)}(x, \mathbf{n}(x)) w_{1}\right)=0,
$$

поскольку условие $(7)$ выполнено для $A(t)$ и расслоения $L(t)$. Отсюда, опять в силу результатов из [29] и гл. 18 и 19 в [28], получаем утверждение предложения сначала для оператора $\mathfrak{A}$, а затем, как следствие, и для его киральной части $\partial / \partial t+A(t)$.

ДокАЗАТЕЛЬСТВо ТЕОРЕмы 2. Без ограничения общности будем предполагать, что $0 \notin \operatorname{Spec}\left(A(0)_{L(0)}\right)$ (в противном случае можно заменить оператор $A(t)$ на $A(t)+\varepsilon$ с малым вещественным $\varepsilon$, что не меняет ни правую, ни левую часть формулы $(12))$.

Также без ограничения общности будем всюду далее считать, что подрасслоение $L(t)$ не зависит от параметра $t, L(t)=L(0) \equiv L, t \in[0,1]$. Действительно, пусть $V(t): E_{Y} \rightarrow E_{Y}-$ семейство унитарных автоморфизмов расслоения $E_{Y}$ такое, что $V(0)=I$ и $L(t)=V(t) L, t \in[0,1]$ (такое семейство легко построить, решая задачу Коши $\dot{V}=[P, \dot{P}] V, V(0)=I$, где $P=P(t)$ - проектор на $L(t)$ в $\left.E_{Y}\right)$. Это семейство продолжается (гомотопией к тождественному отображению по нормальной к границе переменной) до семейства унитарных автоморфизмов $W(t): E \rightarrow E$ такого, что $\left.W(t)\right|_{Y}=V(t)$. Положим

$$
\tilde{A}(t)=W^{-1}(t) A(t) W(t)
$$

тогда, очевидно,

$$
\begin{gathered}
W^{-1}(1) U \tilde{A}(0)\left(W^{-1}(1) U\right)^{-1}=W^{-1}(1) U \tilde{A}(0) U^{-1} W(1)=W^{-1}(1) A(1) W(1)=\tilde{A}(1), \\
W^{-1}(1) U L \\
\equiv W^{-1}(1) U L(0)=W^{-1}(1) L(1)=L(0) \equiv L,
\end{gathered}
$$

т. е. условия вида (9) выполнены для семейства $\{\tilde{A}(t)\}$ и постоянного семейства подпространств $\tilde{L}(t)=L$, если взять автоморфизм $\widetilde{U}=W^{-1}(1) U$. Кроме того,

$$
\operatorname{sf}\left\{A(t)_{L(t)}\right\}=\operatorname{sf}\left\{\tilde{A}(t)_{L}\right\}
$$


в силу подобия операторов $A(t)$ и $\tilde{A}(t)$. Далее, семейство $W(t)$ порождает изоморфизм расслоений $\mathcal{W}: \widetilde{\mathcal{E}} \rightarrow \mathcal{E}$, где расслоение $\widetilde{\mathcal{E}}$ над $X \times S^{1}$, аналогично $\mathcal{E}$, получается из поднятия на $X \times[0,1]$ расслоения $E$ сцеплением по автоморфизму $\widetilde{U}$. Оператор

$$
\mathcal{W}^{-1}\left(\frac{\partial}{\partial t}+A(t)\right)_{\mathcal{L}} \mathcal{W}=\left(\frac{\partial}{\partial t}+\tilde{A}(t)-W^{-1}(t) \frac{\partial W}{\partial t}(t)\right)_{\tilde{\mathcal{L}}}
$$

имеет тот же индекс, что и $(\partial / \partial t+A(t))_{\mathcal{L}}$, и действует в пространстве сечений расслоения $\widetilde{\mathcal{E}}$, удовлетворяющих граничному условию, порожденному подрасслоением $\widetilde{\mathcal{L}}=\left.\mathcal{W}^{-1}\right|_{Y \times S^{1}} \mathcal{L}$, для которого $\widetilde{\mathcal{L}}_{t}=V(t)^{-1} L(t)=L$ при всех $t \in S^{1}$. Наконец, гомотопия

$$
\left(\frac{\partial}{\partial t}+\tilde{A}(t)-\lambda W^{-1}(t) \frac{\partial W}{\partial t}(t)\right)_{\tilde{\mathcal{L}}}, \quad \lambda \in[0,1],
$$

в классе фредгольмовых операторов приводит оператор (16) при $\lambda=0 \mathrm{k}(\partial / \partial t+$ $\tilde{A}(t))_{\widetilde{\mathcal{L}}}$, так что

$$
\operatorname{ind}\left(\frac{\partial}{\partial t}+A(t)\right)_{\mathcal{L}}=\operatorname{ind}\left(\frac{\partial}{\partial t}+\tilde{A}(t)\right)_{\widetilde{\mathcal{L}}}
$$

что вместе с (15) и завершает редукцию к случаю не зависящего от $t$ расслоения $L(t)=L$. Тильду над буквами в дальнейшем опускаем.

Теперь для доказательства нам потребуется вспомогательное семейство операторов на бесконечном цилиндре $X \times \mathbb{R}$. Поднятия расслоений $E$ с $X$ на $X \times \mathbb{R}$ и $L$ с $Y$ на $Y \times \mathbb{R}$ обозначим теми же буквами $E$ и $L$, это не должно привести к путанице. Координату на прямой $\mathbb{R}$ обозначим через $t$. Для $\alpha, \beta \in \mathbb{R}$ введем весовые пространства $L_{\alpha \beta}^{2}(X \times \mathbb{R}, E)$ и $H_{\alpha \beta}^{1}(X \times \mathbb{R}, E)$ сечений $u$ расслоения $E$ с конечной нормой

$$
\|u\|_{0, \alpha \beta}=\left\{\int_{-\infty}^{0}\|u(t)\|_{L^{2}(X, E)}^{2} e^{2 \alpha t} d t+\int_{0}^{\infty}\|u(t)\|_{L^{2}(X, E)}^{2} e^{2 \beta t} d t\right\}^{1 / 2}
$$

и

$$
\begin{aligned}
\|u\|_{1, \alpha \beta}= & \left\{\int_{-\infty}^{0}\left(\left\|\frac{\partial u(t)}{\partial t}\right\|_{L^{2}(X, E)}^{2}+\|u(t)\|_{H^{1}(X, E)}^{2}\right) e^{2 \alpha t} d t+\right. \\
& \left.+\int_{0}^{\infty}\left(\left\|\frac{\partial u(t)}{\partial t}\right\|_{L^{2}(X, E)}^{2}+\|u(t)\|_{H^{1}(X, E)}^{2}\right) e^{2 \beta t} d t\right\}^{1 / 2}
\end{aligned}
$$

соответственно. В частности, $H_{\alpha \beta}^{1}(X \times \mathbb{R}, E) \subset L_{\alpha \beta}^{2}(X \times \mathbb{R}, E)$. Через $\mathfrak{D}_{\alpha \beta}$ обозначим замкнутое подпространство в $H_{\alpha \beta}^{1}(X \times \mathbb{R}, E)$, состоящее из сечений, удовлетворяющих граничным условиям, задаваемым подрасслоением $L$, т. е.

$$
\mathfrak{D}_{\alpha \beta}=\left\{u \in H_{\alpha \beta}^{1}(X \times \mathbb{R}, E): \pi_{L} u=0\right\} .
$$

Пусть $0 \leqslant \theta \leqslant 1$. Положим ${ }^{5)}$

$$
\tau(t, \theta)= \begin{cases}0, & t \leqslant 0 \\ t, & 0 \leqslant t \leqslant \theta \\ \theta, & \theta \leqslant t\end{cases}
$$

5) Здесь, так же как и выше и ниже, при описании конструкции гомотопий во избежание громоздких формул мы опускаем стандартную процедуру сглаживания, устраняющую разрывы производных, в данном случае при $t=0$ и $t=\theta$. 


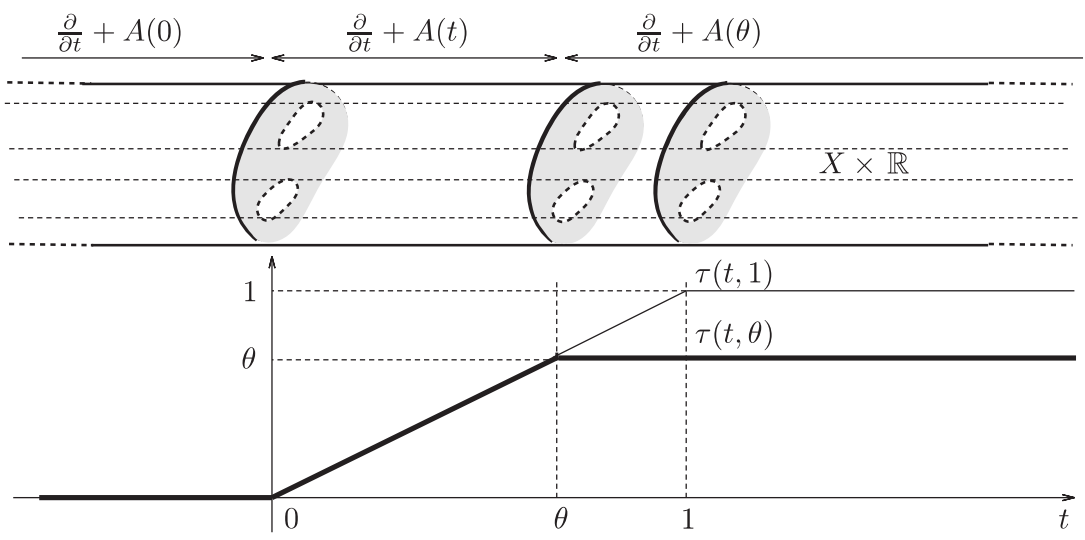

Рис. 4. Оператор $\mathcal{A}(\theta, \gamma)=\partial / \partial t+A(\tau(t, \theta))$ на бесконечном цилиндре $X \times \mathbb{R}$.

Для $\gamma \in \mathbb{R}$ пусть

$$
\mathcal{A}(\theta, \gamma)=\frac{\partial}{\partial t}+A(\tau(t, \theta)): L_{0 \gamma}^{2}(X \times \mathbb{R}, E) \longrightarrow L_{0 \gamma}^{2}(X \times \mathbb{R}, E)
$$

- оператор с областью определения $\mathfrak{D}_{0 \gamma}$ (см. рис. 4). Сформулируем ряд свойств операторов $\mathcal{A}(\theta, \gamma)$.

Лемма 1. Оператор $\mathcal{A}(\theta, \gamma)$ является фредголъмовым при тех $\theta$, при которых $\gamma \notin \operatorname{Spec}\left(A(\theta)_{L}\right), u$ ind $\mathcal{A}(\theta, \gamma)$ - локалъно постоянная функиия от $\theta$ на множестве таких значений $\theta$.

Лемма 2. Если $\gamma, \widetilde{\gamma} \notin \operatorname{Spec}\left(A(\theta)_{L}\right)$ u $\gamma>\widetilde{\gamma}$, mо разность ind $\mathcal{A}(\theta, \widetilde{\gamma})-\operatorname{ind} \mathcal{A}(\theta, \gamma)$ равна числу собственных значений (с учетом кратности) оператора $A(\theta)_{L}$ в интервале $(\widetilde{\gamma}, \gamma)$.

Лemma 3. ind $\mathcal{A}(0,0)=0$.

Лemma 4. ind $\mathcal{A}(1,0)=\operatorname{ind}(\partial / \partial t+A(t))_{\mathcal{L}}$.

Доказательство лемм 1-4 будет дано ниже. Сейчас покажем, что из этих лемм следует утверждение теоремы. Спектральный поток семейства $\left\{A(t)_{L}\right\}$ дается определением 1 для некоторого разбиения $0=t_{0}<t_{1}<\cdots<t_{n+1}=1$ и вещественных чисел $\gamma_{1}, \ldots, \gamma_{n+1}=\gamma_{1}$, причем можно считать, что $\gamma_{1}=0$ (так как мы предположили, что $\left.0 \notin \operatorname{Spec}\left(A(0)_{L}\right)\right)$. Пусть $m_{j}$ - количество собственных значений (с учетом кратности) оператора $A\left(t_{j}\right)_{L}$ в интервале между $\gamma_{j}$ и $\gamma_{j+1}$. Из леммы 1 следует, что оператор $\mathcal{A}\left(\theta, \gamma_{j}\right)$ фредгольмов при $\theta \in\left[t_{j-1}, t_{j}\right]$ и

$$
\text { ind } \mathcal{A}\left(t_{j}, \gamma_{j}\right)-\text { ind } \mathcal{A}\left(t_{j-1}, \gamma_{j}\right)=0, \quad j=1, \ldots, n+1 \text {. }
$$

Согласно лемме 2

$$
\text { ind } \mathcal{A}\left(t_{j}, \gamma_{j+1}\right)-\text { ind } \mathcal{A}\left(t_{j}, \gamma_{j}\right)=m_{j} \operatorname{sign}\left(\gamma_{j}-\gamma_{j+1}\right), \quad j=1, \ldots, n \text {. }
$$


Суммируя равенства (20) и (21) по всем соответствующим $j$, складывая результаты между собой и учитывая лемму 3 и равенство $\gamma_{1}=\gamma_{n}=0$, получаем

$$
\text { ind } \mathcal{A}(1,0)=\text { ind } \mathcal{A}(1,0)-\text { ind } \mathcal{A}(0,0)=\sum_{j=1}^{n} m_{j} \operatorname{sign}\left(\gamma_{j}-\gamma_{j+1}\right)=\operatorname{sf}\left\{A(t)_{L}\right\} .
$$

Остается применить лемму 4.

Докажем теперь леммы 1-4.

ДокАЗАТЕЛЬСтво ЛЕммы 1. Чтобы доказать, что оператор $\mathcal{A}(\theta, \gamma)$ является фредгольмовым, достаточно построить его регуляризатор, т. е. оператор

$$
\mathcal{R}: L_{0 \gamma}^{2}(X \times \mathbb{R}, E) \longrightarrow \mathfrak{D}_{0 \gamma}
$$

такой, что операторы $I-\mathcal{A}(\theta, \gamma) \mathcal{R}$ и $I-\mathcal{R} \mathcal{A}(\theta, \gamma)$ компактны в пространствах $L_{0 \gamma}^{2}(X \times \mathbb{R}, E)$ и $\mathfrak{D}_{0 \gamma}$ соответственно. Это можно сделать стандартным в эллиптической теории методом замораживания коэффициентов (в данном случае по переменной $t$ ). Для этого при фиксированных $\tau \in[0,1], \nu \in \mathbb{R}$ рассмотрим оператор

$$
\frac{\partial}{\partial t}+A(\tau): L_{\nu \nu}^{2}(X \times \mathbb{R}, E) \longrightarrow L_{\nu \nu}^{2}(X \times \mathbb{R}, E) \quad \text { с областью определения } \mathfrak{D}_{\nu \nu} .
$$

Оператор (23) обратим при условии, что $\nu \notin \operatorname{Spec}\left(A(\tau)_{L}\right)$. Обратный оператор, который мы обозначим через $R_{\nu}(\tau)$, задается формулой

$$
\left[R_{\nu}(\tau) u\right](t)=\frac{1}{\sqrt{2 \pi}} \int_{\operatorname{Im} p=\nu} e^{i p t}\left(i p+A(\tau)_{L}\right)^{-1} \widetilde{u}(p) d p, \quad u \in L_{\nu \nu}^{2}(X \times \mathbb{R}, E)
$$

где $\widetilde{u}(p), \operatorname{Im} p=\nu,-$ преобразование Фурье функции $u$ по переменной $t$. Рассмотрим конечное покрытие $\left\{U_{j}\right\}_{j=1}^{s}$ отрезка $[0,1]$ интервалами такими, что $\nu_{j} \notin$ $\operatorname{Spec}\left(A(\tau(t, \theta))_{L}\right)$ при $t \in U_{j}$ для некоторых вещественных чисел $\nu_{j}$, пусть $U_{0}=$ $(-\infty, 0), \nu_{0}=0, U_{s+1}=(1, \infty), \nu_{s+1}=\gamma$ и пусть $1=\sum_{j=0}^{s+1} \psi_{j}^{2}$ - гладкое разбиение единицы, подчиненное покрытию прямой $\mathbb{R}$ множествами $U_{j}, j=0, \ldots, s+1$. Тогда оператор $\mathcal{R}$ можно задать формулой

$$
[\mathcal{R} u](t)=\left.\sum_{j=0}^{s+1} \psi_{j}(t)\left[\left[\mathcal{R}_{\nu_{j}}(\tau)\left(\psi_{j} u\right)\right](t)\right]\right|_{\tau=\tau(t, \theta)} .
$$

(Заметим, что оператор $\mathcal{R}$ корректно определен как оператор из $L_{0 \gamma}^{2}(X \times \mathbb{R}, E)$ в $\mathfrak{D}_{0 \gamma}$, поскольку оператор умножения на $\psi_{j}$ непрерывно действует из $L_{0 \gamma}^{2}(X \times \mathbb{R}, E)$ в $L_{\nu_{j} \nu_{j}}^{2}(X \times \mathbb{R}, E)$ и из $\mathfrak{D}_{\nu_{j} \nu_{j}}$ в $\mathfrak{D}_{0 \gamma} ;$ при $j \neq 0, s+1$ это следует из компактности носителя функции $\psi_{j}$, при $j=0$ - из того, что $\nu_{0}=0$ и функция $\psi_{0}$ равна нулю при $t>0$, а при $j=s+1-$ из того, что $\nu_{s+1}=\gamma$ и функция $\psi_{s+1}$ равна нулю при $t<0$.) Теперь непосредственное вычисление показывает, что

$$
\begin{aligned}
& \mathcal{A}(\theta, \gamma) \mathcal{R} u(t)= \\
& =u(t)+\left.\sum_{j=0}^{s+1}\left[\left[\left(\frac{\partial \psi_{j}}{\partial t}(t) \mathcal{R}_{\nu_{j}}(\tau)+\psi_{j}(t) \frac{\partial \tau}{\partial t}(t, \theta) \frac{\partial \mathcal{R}_{\nu_{j}}}{\partial \tau}(\tau)\right)\left(\psi_{j} u\right)\right](t)\right]\right|_{\tau=\tau(t, \theta)} .
\end{aligned}
$$


Поскольку функции $\left(\partial \psi_{j} / \partial t\right)(t)$ и $(\partial \tau / \partial t)(t, \theta)$ финитны, стандартные утверждения о вложениях пространств Соболева показывают, что второе слагаемое в правой части задает компактный оператор в $L_{0 \gamma}^{2}(X \times \mathbb{R}, E)$. Аналогичным образом рассматривается и композиция $\mathcal{R} \mathcal{A}(\theta, \gamma)$.

Локальное постоянство индекса оператора $\mathcal{A}(\theta, \gamma)$ как функции от $\theta$ следует из того, что этот оператор непрерывно зависит от $\theta$ в операторной норме как оператор из $\mathfrak{D}_{0 \gamma}$ в $L_{0 \gamma}^{2}(X \times \mathbb{R}, E)$.

ДоказАТЕЛьство лЕммы 3. Утверждение леммы - это частный случай обратимости оператора (23) при $\nu=0$ и $\tau=0$.

Доказательство лемм 2 и 4 опирается на метод локализации (принцип локальности индекса; см. теорему 4.10 в [26], а также [23]-[25] и цитированную там литературу). Для наших целей сформулируем утверждение теоремы 4.10 из [26] в простейшем частном случае.

Пусть $N_{1}, N_{2} \subset M$ - непересекающиеся замкнутые подмножества многообразия $M$, и пусть $D: \mathcal{H}_{1} \longrightarrow \mathcal{H}_{2}$ - ограниченный фредгольмов оператор, действующий в некоторых гилъбертовых пространствах сечений расслоений над многообразием $M$. Далее, пусть к: $M \rightarrow[0,1]$ - гладкое отображение такое, что $N_{1} \subset f^{-1}(0)$ и $N_{2} \subset f^{-1}(1)$, и пусть $\mathcal{C} \subset C^{\infty}(M)$ - некоторая подалгебра алгебры гладких функций на $M$, состоящая из функций, постоянных на $N_{1}$ и на $N_{2}$, и содержащая все функиии вида $\psi(x)=\varphi(\varkappa(x))$, где $\varphi$ - гладкая функция на отрезке $[0,1]$. Предположим, что коммутатор оператора $D$ с оператором умножения на любую функцию из $\mathcal{C}$ компактен. Тогда приращения индекса, возникающие при изменении оператора $D$ на $N_{1}$ и $N_{2}$ с сохранением фредгольмовости и компактности коммутаторов ${ }^{6)}$, независимы:

$$
\Delta_{N_{1} \sqcup N_{2}}=\Delta_{N_{1}}+\Delta_{N_{2}}
$$

где $\Delta_{N_{1}}$ - приращение индекса при изменении оператора только на $N_{1}, \Delta_{N_{2}}$ - приращение индекса при изменении оператора только на $N_{2}, \Delta_{N_{1} \sqcup N_{2}}$ - приращение индекса при одновременном изменении оператора и на $N_{1}$, и на $N_{2}$.

Практическое применение метода локализации к доказательству лемм 2 и 4 выглядит следующим образом. Мы хотим вычислить, как изменяется индекс некоего оператора $D$ при заданном его изменении на множестве $N_{1}$, но подсчитать такое приращение сложно из-за сложной структуры оператора $D$ вне $N_{1}$. Изменим оператор $D$ на некотором множестве $N_{2}$, не пересекающемся с $N_{1}$, так, чтобы получился оператор $\widetilde{D}$ более простой структуры, приращение индекса которого при рассматриваемом изменении на $N_{1}$ уже удается подсчитать. Это приращение совпадает с требуемым приращением для исходного оператора.

ДокАЗАТЕЛЬство леммы 2. В качестве многообразия $M$ возьмем $X \times \mathbb{R}$, в качестве множества $N_{1} \subset M-$ множество $\{t \geqslant 2\}$, в качестве множества $N_{2} \subset M-$ множество $\{t \leqslant 1\}$, а в качестве алгебры функций $\mathcal{C}$ - алгебру бесконечно дифференцируемых функций $\varphi(t)$ от переменной $t \in \mathbb{R}$, постоянных на $N_{1}$ и на $N_{2}$. Исходный

\footnotetext{
6) Изменение может затрагивать не только сам оператор, но и пространства, в которых он действует, и даже само многообразие (например, отрезание одних частей и приклеивание других); все эти изменения должны происходить строго внутри соответствующего множества $N_{j}$, т. е. на $M \backslash N_{j}$ все должно оставаться неизменным.
} 
оператор $D$ - это оператор $A(\theta, \gamma)$, который будем рассматривать как ограниченный фредгольмов оператор в пространствах

$$
D=A(\theta, \gamma): \mathfrak{D}_{0 \gamma} \longrightarrow L_{0 \gamma}^{2}(X \times \mathbb{R}, E) ;
$$

требуется подсчитать приращение его индекса при замене $\gamma$ на $\widetilde{\gamma}$. Заметим, что коммутатор оператора $(25)$ с гладкой функцией $\varphi \in \mathcal{C}$ есть оператор умножения на финитную функцию $\varphi^{\prime}(t)$, который компактен как оператор из $\mathfrak{D}_{0 \gamma}$ в $L_{0 \gamma}^{2}\left(X \times \mathbb{R}^{1}, E\right)$, так что мы находимся как раз в ситуации, в которой применим метод локализации. Замена $\gamma$ на $\widetilde{\gamma}$ изменяет оператор $D$ только на множестве $N_{1}$ (выражение, задающее оператор, и граничные условия остаются прежними, но пространства, в которых он действует, меняются, причем это изменение касается только допустимого роста функций при $t \rightarrow+\infty$, т. е., в частности, сужение этих пространств на $M \backslash N_{1}$ вообще не изменяется). Подменим теперь оператор $D$ оператором

$$
\widetilde{D}=\frac{\partial}{\partial t}+A(\theta): \mathfrak{D}_{\gamma \gamma} \longrightarrow L_{0 \gamma}^{2}(X \times \mathbb{R}, E) .
$$

Этот оператор отличается от $D$ (как дифференциальным выражением, так и пространствами, в которых он действует) только на подмножестве $N_{2}$. Таким образом, достаточно посчитать приращение индекса этого оператора при таком же, как у $D$, изменении на $N_{1}$. Оператор $\widetilde{D}$ обратим, так что ind $\widetilde{D}=0$. Его изменение на $N_{1}$ приводит к оператору

$$
\frac{\partial}{\partial t}+A(\theta): \mathfrak{D}_{\gamma \widetilde{\gamma}} \longrightarrow L_{\gamma \widetilde{\gamma}}^{2}(X \times \mathbb{R}, E)
$$

осталось, таким образом, подсчитать индекс последнего. Для этого удобно трактовать оператор (27) как неограниченный фредгольмов оператор в $L_{\gamma \widetilde{\gamma}}^{2}(X \times \mathbb{R}, E)$ с областью определения $\mathfrak{D}_{\gamma \widetilde{\gamma}}$. Сопряженный оператор тогда имеет вид $-\partial / \partial t+A(\theta)$ и действует в сопряженном пространстве $L_{-\gamma,-\widetilde{\gamma}}^{2}(X \times \mathbb{R}, E)$ с областью определения $\mathfrak{D}_{-\gamma,-\widetilde{\gamma}}$. Элементы ядра оператора (27) должны иметь вид $v(x) e^{-\lambda t}$, где $\lambda$ - собственные значения оператора $A(\theta)_{L}$, а $v(x)$ - соответствующие собственные функции. Из условия принадлежности этих элементов весовому пространству $L_{\gamma \widetilde{\gamma}}^{2}(X \times \mathbb{R}, E)$ вытекает, что $\widetilde{\gamma}<\lambda<\gamma$. Таким образом, размерность ядра равна числу собственных значений (с учетом кратности) оператора $A(\theta)_{L}$ в интервале $(\widetilde{\gamma}, \gamma)$. Элементы ядра сопряженного оператора должны иметь вид $v(x) e^{\lambda t}$ и принадлежать весовому пространству $L_{-\gamma,-\widetilde{\gamma}}^{2}(X \times \mathbb{R}, E)$. Отсюда вытекает, что $\gamma<\lambda<\widetilde{\gamma}$, но это невозможно, ибо $\gamma>\widetilde{\gamma}$. Итак, ядро сопряженного оператора тривиально, индекс оператора (27) совпадает с размерностью его ядра, и мы получаем утверждение леммы.

ДокАзАТЕЛЬство лЕммы 4. Нам необходимо доказать равенство индексов операторов

$$
\mathcal{A}(1,0): \mathfrak{D}_{00} \longrightarrow L^{2}(X \times \mathbb{R}, E)
$$

и

$$
\left(\frac{\partial}{\partial t}+A(t)\right)_{\mathcal{L}}: \mathfrak{D}_{\mathcal{L}} \longrightarrow L^{2}\left(X \times S^{1}, \mathcal{E}\right) .
$$

Будем считать (этого можно всегда добиться гомотопией), что $A(t)=A(1)$ при $t \geqslant 1-2 \varepsilon$ и $A(t)=A(0)$ при $t \leqslant 2 \varepsilon$ для некоторого фиксированного $\varepsilon>0$. Положим

$$
N_{1}=\{t \in(-\infty, \varepsilon] \cup[1-\varepsilon, \infty)\}, \quad N_{2}=\{t \in[2 \varepsilon, 1-2 \varepsilon]\} .
$$


В качестве алгебры функций $\mathcal{C}$ снова возьмем алгебру бесконечно дифференцируемых функций $\varphi(t)$ от переменной $t$, постоянных на $N_{1}$ и на $N_{2}$. Оператор (29) получается из оператора (28) следующим изменением на множестве $N_{1}$ : бесконечные "полуцилиндры" $X \times(-\infty, 0)$ и $X \times(1, \infty)$ отрезаются и выбрасываются, а торцы $X \times\{0\}$ и $X \times\{1\}$ оставшегося произведения $X \times[0,1]$ склеиваются, причем расслоение $E$ склеивается в расслоение $\mathcal{E}$ сцеплением по автоморфизму $U:\left.\left.E\right|_{t=0} \longrightarrow E\right|_{t=1}$.

Итак, мы должны показать, что приращение индекса при таком изменении оператора на $N_{1}$ равно нулю. Для этого подменим оператор (28) оператором

$$
\mathcal{A}(0,0): \mathfrak{D}_{00} \longrightarrow L^{2}(X \times \mathbb{R}, E)
$$

Оператор (30) будет отличаться от оператора (28) только на $N_{2}$, если записать его в эквивалентной форме

$$
\mathcal{A}(0,0)=\left\{\begin{array}{lll}
\frac{\partial}{\partial t}+A(0) & \text { при } & t \leqslant \frac{1}{2}, \\
\frac{\partial}{\partial t}+A(1) & \text { при } & t \geqslant \frac{1}{2},
\end{array}\right.
$$

где подразумевается, что расслоение $E$ при $t=1 / 2$ склеено сцеплением по автоморфизму

$$
U^{-1}:\left.\left.E\right|_{t=1 / 2+0} \longrightarrow E\right|_{t=1 / 2-0} .
$$

(Переход от (30) к (31) есть, по существу, не что иное, как просто запись оператора $\mathcal{A}(0,0)$ при $t \geqslant 1 / 2$ в "новых координатах" в слоях расслоения $E$.)

Применим теперь к оператору (30), записанному в форме (31), то же изменение на $N_{1}$, что было ранее применено к оператору (28). Полученный в результате оператор на $X \times S_{1}$ имеет вид (31), причем расслоение, в котором он действует, получено из $E$ сцеплением по автоморфизмам

$$
U:\left.\left.E\right|_{t=0} \longrightarrow E\right|_{t=1}, \quad U^{-1}:\left.\left.E\right|_{t=1 / 2+0} \longrightarrow E\right|_{t=1 / 2-0} .
$$

Нетрудно видеть, что это расслоение изоморфно поднятию расслоения $E$ на $X \times$ $S_{1}$, а сам полученный оператор есть не что иное, как $(\partial / \partial t+A(0))_{L}$, и его индекс равен нулю (так как он инвариантен относительно вращений вдоль $S^{1}$ ). Индекс оператора (28) также равен нулю (он обратим), так что приращение индекса равно нулю.

ДокАЗАТЕЛЬСтво теоремы 1. Теорема 2 показывает, что для спектрального потока семейства (2) справедлив принцип локализации: любые модификации, которые мы применим к рассматриваемому оператору Дирака (1) в плоской области $X$ и к входящей в граничные условия функции $B$, автоматически поднимаются на $X \times S_{1}$, превращаясь там в модификации оператора $\partial / \partial t+D_{t}$, а для них справедлив принцип локальности индекса (см. теорему 4.10 в [26]), который в силу теоремы 2 равен спектральному потоку.

Принцип локализации позволяет разбивать область с дырами на части, содержащие меньшее число дыр. Покажем это на примере. На рис. 5 слева показана область $X$ с двумя дырами. Покажем, что вычисление спектрального потока для семейства операторов Дирака с локальными краевыми условиями в такой области 

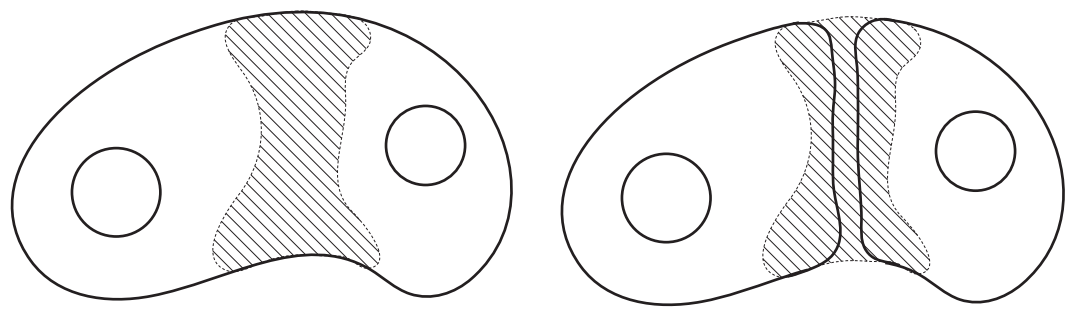

Рис. 5. Разрезание на части области с дырами.
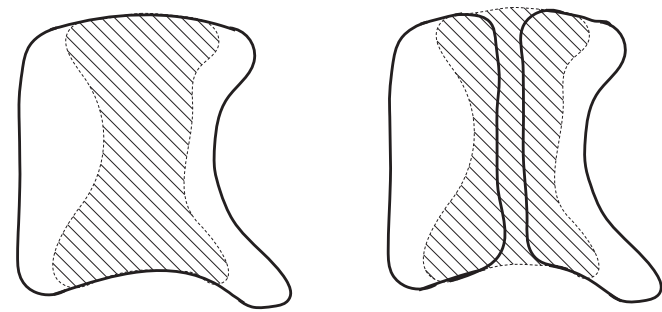

Рис. 6. Разрезание на части области без дыр.

сводится к вычислению для областей с одной дырой. Действительно, пусть $N_{1}-$ множество, заштрихованное на рис. 5 слева, а $N_{2}$ - дополнение к малой окрестности множества $N_{1}$. Изменим область внутри $N_{1}$, как показано на рис. 5 справа, так, что из исходной области получаются две области с гладкой границей. Функция $B(x)$, входящая в граничные условия, может быть продолжена непрерывным образом на возникшие внутри области $N_{1}$ новые участки границы как не обращающаяся в нуль вещественная функция, так как ее знак на всей внешней границе исходной области один и тот же. Таким образом, область распалась на две не связанные между собой части, и чтобы доказать, что спектральный поток для семейства операторов Дирака в исходной области совпадает с суммой спектральных потоков, отвечающих двум вновь возникшим областям, нужно показать, что приращение спектрального потока при такой модификации области равно нулю. Для доказательства воспользуемся методом локализации. Изменим исходное семейство, изменив область в $N_{2}$ (так, что получится область, показанная на рис. 6 слева) и продолжая $B(x)$ непрерывным образом на возникший внутри $N_{2}$ новый участок границы как не обращающуюся в нуль вещественную функцию. Для нового семейства спектральный поток и до, и после модификации, показанной на рис. 6, равен нулю, поскольку области на этом рисунке стягиваемы и калибровочное преобразование $\mu$ в них гомотопно тривиальному. Итак, разрезая область на части, можно свести дело к случаю области с одной дырой, а для таких областей формула (3) была доказана в работе [20]. (Разумеется, можно было бы доказать ее и непосредственно с помощью теоремы 2, но необходимости в этом нет, и мы не приводим соответствующие выкладки за недостатком места.) 


\section{5. ЗАКЛЮЧЕНИЕ}

Обсудим возможную физическую реализацию ненулевого спектрального потока. Начнем со случая графена. Следует иметь в виду, что в графене имеется две дираковские подсистемы электронов (две долины) и, вообще говоря, рассеяние на границах образца смешивает долины [7], [31]. Исключение составляет случай, когда при приближении к границе достаточно плавно открывается энергетическая щель в электронном энергетическом спектре. Такая ситуация естественно имеет место при химической функционализации границ, которая модифицирует электронную структуру в достаточно большой области пространства [32]. В результате рассеянием между долинами можно пренебречь, и мы имеем граничное условие в задаче (1), впервые предложенное Берри и Мондрагоном [19]. Детальный микроскопический вывод граничного условия, начинающийся с дискретной решеточной модели, дан в работе [31] (см. также главу 5 в [7] и цитированную там литературу). Знак константы $B$ при этом определяется знаком появляющегося массового члена в уравнении Дирака, а это, в свою очередь, определяется тем, как именно расположатся на границе химические группы. Можно надеяться, что в части образцов (если приготовить графеновое кольцо) знаки константы $B$ будут разными на внутреннем и внешнем краях кольца, что необходимо для ненулевого спектрального потока. Такой случай, однако, трудно реализовать контролируемым образом. Видимо, больше возможностей предоставляют топологические изоляторы. Во-первых, двумерная дираковская система реализуется на поверхности трехмерных топологических изоляторов, таких как $\mathrm{Bi}_{2} \mathrm{Se}_{3}$, причем дираковский конус в этом случае может быть один [8], [9]. Для открытия щели (появления массового члена) нужно покрыть поверхность магнитным слоем, причем знак массы определяется направлением намагниченности [8], [9]. Это открывает возможность манипулирования знаком константы $B$. Во-вторых, двумерные безмассовые дираковские электроны могут быть реализованы в слое бесщелевого полупроводника $\mathrm{HgTe}$, зажатого между двумя слоями $\mathrm{CdTe,} \mathrm{при} \mathrm{некоторой} \mathrm{критической} \mathrm{толщине} \mathrm{слоя} \mathrm{[8],} \mathrm{[9].} \mathrm{Недавно} \mathrm{такая} \mathrm{возможность}$ была продемонстрирована экспериментально [33]. Если толщина слоя плавно меняется в пространстве, отклоняясь от критического значения, можно реализовать как положительные, так и отрицательные значения массового члена. В данный момент эта возможность реализации рассмотренной нами ситуации ненулевого спектрального потока выглядит самой привлекательной.

Благодарности. Исследования В.Е. Назайкинского были поддержаны РФФИ (грант № 11-01-00973). Авторы признательны С. Ю. Доброхотову и А. И. Шафаревичу за внимание к работе и полезные обсуждения.

\section{Список литературы}

[1] N. D. Mermin, Rev. Modern Phys., 51:3 (1979), 591-648.

[2] D. J. Thouless, M. Kohmoto, M. P. Nightingale, M. den Nijs, Phys. Rev. Lett., 49:6 (1982), 405-408.

[3] J. Bellisard, A. van Elst, H. Schulz-Baldes, J. Math. Phys., 35:10 (1994), 5373-5451, arXiv: cond-mat/9411052.

[4] F. Wilczek, Fractional Statistics and Anyon Superconductivity, World Scientific, Singapore, 1990. 
[5] G. E. Volovik, The Universe in a Helium Droplet, International Series of Monographs on Physics, 117, Oxford Univ. Press, Oxford, 2003.

[6] M. A. H. Vozmediano, M. I. Katsnelson, F. Guinea, Phys. Rep., 496:4-5 (2010), 109-148, arXiv: 1003.5179.

[7] M.I. Katsnelson, Graphene. Carbon in Two Dimensions, Cambridge Univ. Press, Cambridge, 2012.

[8] M. Z. Hasan, C. L. Kane, Rev. Modern Phys., 82:4 (2010), 3045-3067, arXiv: 1002.3895.

[9] X.-L. Qi, S.-C. Zhang, Rev. Modern Phys., 83:4 (2011), 1057-1110.

[10] M. F. Atiyah, I. M. Singer, Bull. Amer. Math. Soc., 69 (1963), 422-433.

[11] N. B. Kopnin, Rep. Prog. Phys., 65:11 (2002), 1633-1678.

[12] Y. Aharonov, D. Bohm, Phys. Rev., 115:3 (1959), 485-491.

[13] S. Olariu, I. I. Popescu, Rev. Modern Phys., 57:2 (1985), 339-436.

[14] Y. Avishai, Y. Hatsugai, M. Kohmoto, Phys. Rev. B, 47:15 (1993), 9501-9512.

[15] P. Recher, B. Trauzettel, A. Rycerz, Ya. M. Blanter, C. W. J. Beenakker, A. F. Morpurgo, Phys. Rev. B, 76:23 (2007), 235404, 6 pp.

[16] R. Jackiw, A. I. Milstein, S.-Y. Pi, I. S. Terekhov, Phys. Rev. B, 80:3 (2009), 033413, 3 pp., arXiv: 0904.2046.

[17] M. I. Katsnelson, Europhys. Lett., 89:1 (2010), 17001, 3 pp., arXiv: 0911.3263.

[18] M. F. Atiyah, V. K. Patodi, I. M. Singer, Math. Proc. Cambridge Philos. Soc., 79:1 (1976), 71-99.

[19] M. Berry, R. J. Mondragon, Proc. Roy. Soc. London Ser. A, 412:1842 (1987), 53-74.

[20] M. Prokhorova, The spectral flow for Dirac operators on compact planar domains with local boundary conditions, arXiv: 1108.0806.

[21] M.F. Atiyah, R. Bott, "The index problem for manifolds with boundary", Proceedings of the Bombay Colloquium on Differential Analysis (Bombay, India, January 7-14, 1964), Oxford Univ. Press, Oxford, 1964, 175-186.

[22] L. Hörmander, The Analysis of Linear Partial Differential Operators. III, Grundlehren der Mathematischen Wissenschaften [Fundamental Principles of Mathematical Sciences], 274, Springer, Berlin, 1985.

[23] В. Е. Назайкинский, Б. Ю. Стернин, Докл. РАН, 370:1 (2000), 19-23.

[24] В. Е. Назайкинский, Б. Ю. Стернин, Функи. анализ и его прил., 35:2 (2001), 37-52.

[25] V. Nazaikinskii, B. Sternin, Abstr. Appl. Anal., 2006 (2006), 98081, 16 pp.

[26] V.E. Nazaikinskii, A. Savin, B.-W. Schulze, B. Sternin, Elliptic Theory on Singular Manifolds, Differential and Integral Equations and Their Applications, 7, CRC Press, Boca Raton, FL, 2006.

[27] B. Booß-Bavnbek, M. Lesch, J. Phillips, Canad. J. Math., 57:2 (2005), 225-250.

[28] B. Booß-Bavnbek, K.P. Wojciechowski, Elliptic Boundary Problems for Dirac Operators, Birkhäuser, Boston, MA, 1993.

[29] J. Brüning, M. Lesch, J. Funct. Anal., 185:1 (2001), 1-62.

[30] B. Booß-Bavnbek, M. Lesch, C. Zhu, J. Geom. Phys., 59:7 (2009), 784-826, arXiv: 0803.4160 .

[31] A. R. Akhmerov, C. W. J. Beenakker, Phys. Rev. B, 77:8 (2008), 085423, 10 pp., arXiv: 0710.2723 .

[32] D. W. Boukhvalov, M. I. Katsnelson, Nano Lett., 8:12 (2008), 4373-4379, arXiv: 0807.3855.

[33] B. Büttner, C. X. Liu, G. Tkachov, E. G. Novik, C. Brune, H. Buhmann, E. M. Hankiewicz, P. Recher, B. Trauzettel, S.C. Zhang, L. W. Molenkamp, Nature Physics, 7:5 (2011), 418-422, arXiv: 1009.2248. 